\title{
Management of Femur Refractures in a 3-Year-Old Female with Myelomeningocele. A Case Report and Review of Literature
}

ISSN: 2576-8875

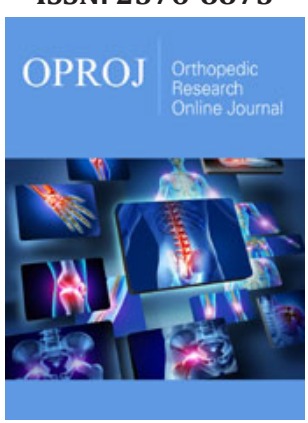

*Corresponding author: Elisa LópezDolado, Rehabilitation Department and Laboratory of Interfaces for Neural Repair (LINER), Hospital Nacional de Parapléjicos, Spain

Submission: 㓶 December 13, 2020

Published: 制December 18, 2020

Volume 7 - Issue 5

How to cite this article: Raquel Madroñero-Mariscal, Elisa LópezDolado*. Management of Femur Refractures in a 3-Year-Old Female with Myelomeningocele. A Case Report and Review of Literature. Ortho Res Online J. 7(5). OPROJ. 000673. 2020. DOI: 10.31031/OPROJ.2020.07.000673

Copyright@: Elisa López-Dolado, This article is distributed under the terms of the Creative Commons Attribution 4.0 International License, which permits unrestricted use and redistribution provided that the original author and source are credited.

\section{Raquel Madroñero-Mariscal ${ }^{1}$ and Elisa López-Dolado ${ }^{2,3,4 *}$}

${ }^{1}$ Physiatrist MD, Laboratory of Interfaces for Neural Repair (LINER), Hospital Nacional de Parapléjicos, Toledo, Spain

${ }^{2}$ Staff Physiatrist, Rehabilitation Department and Laboratory of Interfaces for Neural Repair (LINER), Hospital Nacional de Parapléjicos, Toledo, Spain

${ }^{3}$ Associate Professor, Department of Medicine and Medical Specialities, School of Medicine, Universidad de Alcalá (UAH), Spain

${ }^{4}$ Principal Investigator, Laboratory of Interfaces for Neural Repair, Hospital Nacional de Parapléjicos, Toledo, Spain

\begin{abstract}
Pathological fractures affecting lower limbs in patients with myelomeningocele have a high prevalence and need to be suspected though mild symptoms such as swelling, local redness, or local hyperthermia without pain, due to the lack of sensation of these patients or other systemic factors are presented. These fractures can affect different parts of the bone: metaphysis, diaphysis, or physis. It has been described different details in mechanism of injury, clinical presentation, treatment, and possible complications, depending on the affected site. In this case report, we present a 3-year-old female who has suffered 3 femur fractures in a period of 13 months. We analyze retrospectively the management of this case for formulating recommendations regarding her clinical evolution and the current published data.
\end{abstract}

Keywords: Myelomeningocele; Pathological fractures; Refractures; Metaphysis; Diaphysis; Bone demineralization; Osteoporosis; Bisphosphonates

Abbreviations: PF: Pathological Fractures; MMC: Myelomeningocele; SCI: Spinal Cord Injury; DXA: Dual X-ray Absorptiometry

\section{Introduction}

Pathological Fractures (PF) affecting lower limbs have a prevalence ranging from $9 \%$ to $20 \%$ in children with myelomeningocele (MMC) [1]. It has been supported that the level of the injury involved has a great importance. On the one hand, the upper the level of the injury, the higher the risk of fracture, so thoracic-level injuries have 6 times higher risk of bone fracture, when comparing with sacral-level injuries [1]. On the other hand, Lock and Aronson [2] support that the localization of thoracic-level injuries usually involves femur fractures, while lumbar-level injuries mostly involve tibia fractures [2]; while Aliatakis et al. [3] find in their retrospective study of 210 patients that age at fracture has a stronger correlation with the fracture site than the level of the injury, being the fracture of the femur the most frequent one in the first five years of age and tibia and foot fractures after this age [3]. These fractures can affect different parts of the bone: metaphysis, diaphysis, or physis. It has been described different details in mechanism of injury, clinical presentation, treatment, and possible complications, depending on the affected site of the bone fracture.

It is well known that after Spinal Cord Injury (SCI), it occurs pathological changes in bones and muscles below the neurological level of injury, such as a bone demineralization, muscle atrophy and increased adiposity, that lead to suffer osteopenia and osteoporosis, and therefore it increases the risk of bone fracture [4]. In metaphyseal and diaphyseal fractures, it can rarely, 
but possibly be found a history of minor trauma [5], while physeal fractures are usually associated with repeated forces of stress that are thought to impact this growing part of the bone during walking $[5,6]$, with no history of trauma maybe because of not wearing a suitable orthosis. Taking into account the lack of sensibility due the injury, these patients will not present pain and the only symptoms which may appear are swelling, local redness, local hyperthermia and sometimes high leukocyte count and high temperature, being the last two symptoms specially observed in case of metaphyseal and diaphyseal fractures, which can simulate an osteomyelitis or sarcoma [7-9]. Physeal fractures, occur more frequently unnoticed, with no systemic symptoms and their diagnosis is usually delayed [6]. The treatment of choice has been discussed in the literature and, to our best knowledge, no agreement has been reached yet. There are some published data that support that a conservative treatment management for all these fractures is the best option, even in case of refractures [5]. Others, nevertheless, support that a surgical procedure is required in some cases such as femoral neck and epiphyseal fractures [7], or in case of refractures [1]. There are also authors that do not find differences between conservative and surgical treatment for increasing the risk of refracture [3].

In this case report we present a 3-year-old female who has suffered 3 femur fractures in a period of 13 months. We analyze retrospectively the management of this case for formulating recommendations regarding her clinical evolution and the current published data.

\section{Case Presentation}

A 2-year-old girl, with history of thoracolumbar MMC and, subsequently, flaccid paraplegia, thoracolumbar scolisis, coxa valga and hip instability, developed swelling in left knee without pain, fever, worsening of general condition or discomfort with the palpation of the femur and knee joint, which, however, crackled with the mobilization. The patient had not suffered any obvious trauma. Plain antero-posterior and lateral X-ray showed a nondisplaced, well aligned fracture of the distal femoral metaphysis (Figure 1a $\& 1$ b), so conservative treatment was decided by immobilization with an inguinopedic cast. After 4 weeks, a new X-ray showed incomplete callus formation (Figure 1c), so the immobilization was extended two more weeks, after which, appropriate healing was achieved, although the bones exhibited cortical thinning and loss of bone trabeculation in the metaphysis, suggestive of osteoporosis. After 8 weeks, load over both lower limbs was resumed, with the help of a standing orthosis to guarantee a safe and adequate joints control during the loading. In a blood test, vitamin D deficiency (25- hydroxyvitamin $\mathrm{D}=21 \mathrm{ng} / \mathrm{mL}$ ) was found, accompanied with an increased urine calcium excretion, so calcium and vitamin D supplements were prescribed (Figure 1d \& 1e).
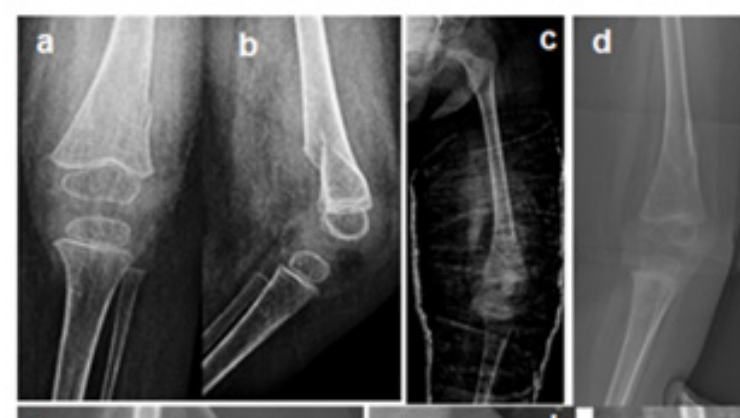

e
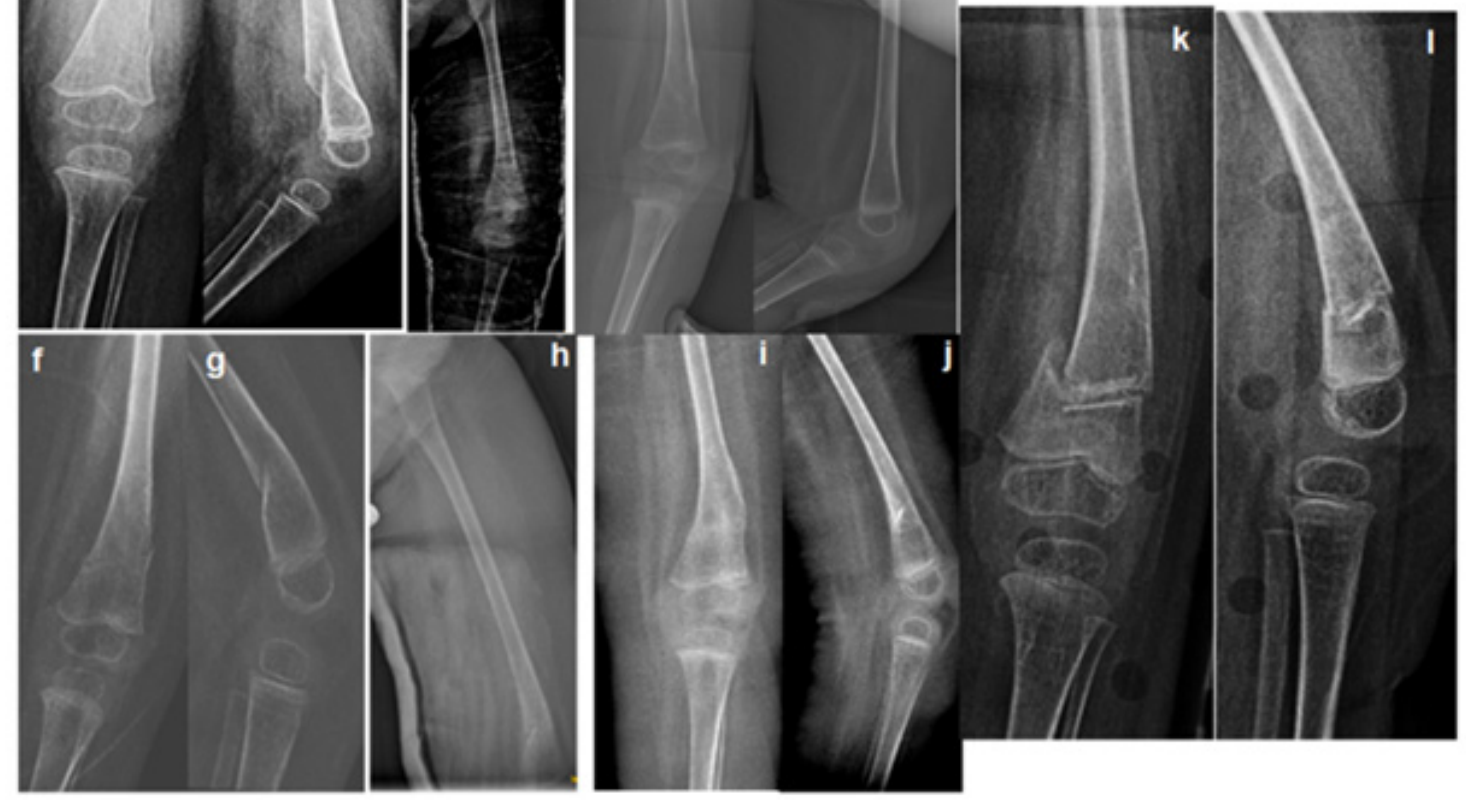

Figure 1: Serial plain X-rays of the sustained metaphyseal femur fracture and the subsequets. Initial fracture lines in antero-posterior a) and lateral b) proyections, incomplete consolidation after 4 weeks by cast immobilization c) and fracture consolidation after 6 weeks, antero-posterior d) and lateral e) proyections respectively. Second fracture, that is to be the first refracture in antero-posterior f) and lateral g) proyections. Control after 4 weeks h) and antero-

posterior d) and lateral e) proyections of fracture consolidation at 6 weeks. Third and last metaphyseal femur fracture, that is to be the second refracture in antero-posterior k) and lateral l) proyections.

Two months after the restarting of the standing, once again, swelling in the same left knee was discovered. Plain X-ray confirmed a new metaphyseal femur fracture just over the previous fracture callus (Figure 1f \& $1 \mathrm{~g}$ ). As also this time the fracture line 
was neat and the bone fragments were not displaced, conservative approach with inguinopedic cast was chosen. A z score -2 was detected in the femur dual X-ray absorptiometry (DXA). To improve the bone modelling bisphosphonates were proposed, but due to an intercurrent episode of urinary tract infection, their administration was temporarily postponed. The cast was removed after 6 weeks (Figure 1i \& 1j) and the loading through a standing orthosis recommenced after 9 weeks. Vitamin D and calcium supplements were maintained to reach a level of 25- hydroxyvitamin D up to 30ng/mL.

Almost a year after the first refracture, a new finding of mild swelling coexisting with left knee crackled with mobilizations made us suspect a second refracture of the former callus, that was confirmed in the X-ray (Figure 1k \& 1l).

\section{Discussion}

In the present work, a thorough description of the management of three femur PF in a 3-year-old female patient with MMC, and a review of the literature are presented.

Classic studies such as Kumar et al. [5], recommend a conservative treatment with a plaster immobilization to manage all these fractures, for 4 weeks in case of metaphyseal and/or diaphyseal fractures and longer periods of immobilization in case of physeal ones [5]. However, later studies like Akbar et al. [1], agree with treating conservatively all these fractures at the initial period, but they support that surgery is needed in case of refracture [1]. Other authors like Pfeil et al. [7] recommend conservative treatment except for femoral neck and epiphysial fractures, which should be initially operated with transcutaneous Kirschner wiring [7], in order to avoid extended immobilization periods, which could increase the bone disuse, as well as osteoporosis appearance and the consequent risk of refracture. Aliatakis et al. [3] did not show differences between conservative and surgical treatments for increasing the risk of refracture, but they found that iatrogenic immobilization is one of the most important risk factors for refractures [3]. Although surgery is not usually the initial chosen option, there are some reported cases such as D'Andrea [10], which show a newborn infant with MMC treated with an external fixator as the first elected treatment, with no posterior complications after 2 years of follow up [10]. In our diaphyseal and metaphyseal fracture case, the initial treatment was conservative, according to the most current published data, but the period of immobilization was longer than expected, taking 6 weeks for the first fracture and also for the first refracture, probably due to the lack of callus formation (Figure 1c) and the development of a concomitant urinary tract infection, which delayed fracture review and required hospitalization, respectively. This resulted in a longer period of no weight bearing, which may have possibly induced the first and second refractures, so the authors of the present case report suggest that surgery may have been considered as the initial treatment option, independently of the affected physeal part of the femur. It should be taken into account that in the daily clinical practice, patients frequently develop unexpected concomitant complications or delayed evolution, which could make longer the immobilization period, as it happened in our case. Therefore, the authors highlight the previous ability of walking as the leading feature to decide surgical initial treatment. Even though in nonambulator patients, the osteopenia and/or osteoporosis induce refractures when long immobilization periods are added as well, it seems to be even more important to ascertain the prevention of this bone deterioration in walker patients that will require an optimal bone recovery, to be able to restore their walking function without risk of refracture, especially at the initial loading period time, which seems to appear as the riskier refracture period. In this situation, an optimal equilibrium between a protective immobilization period and a prompt weight bearing onset should have been achieved to obtain the proper remodeling of the bones originated by the new distribution of the loading forces.

Another important fact is that it should have been considered to start bisphosphonates as pamidronate or zoledronic acid $[1,11,12]$ earlier, as the osteoporosis is certainly causing a great impact in this case, though surgery treatment was rejected to be the first chosen option to treat the first refracture mainly because of a fragile quality of bone. In this way, even though calcium and vitamin D was administered, perhaps if bisphosphonates have been administered as well, surgery treatment for first refracture would have been considered the elected option. There are some other conditions that may have taken part in the increased risk of refracture such as the level of the injury. Aliatakis et al. [3], explain that the risk of refracture is associated with the lesion site, being the thoracic level the riskier one. Also, patients with more risk of refracture are the younger ones (before 6 years old) as it occurs in our case. Moreover, this population has more risk of refractures at shorter interval periods of time when comparing with older children [3]. These last features may have made consider surgery as the first treatment option as well, because it could have provided a quicker and definitive solution. In addition, the surgical approach of this case could preserve the growth cartilages, not affecting the normal growing process of the child.

Another important fact is that, as these fractures usually occur without trauma, it should be considered that orthosis may play a dual function in these patients. On the one hand, its use increases the ability to walk as they help in other neurological disorders, and in the other hand, its use also may play a protective effect for preventing the risk of fracture, because its principal function entails to establish the optimal alignment, position of the joints and distribution of the forces of charges during motion and walking. Therefore, the authors pretend to make pause to think about the necessity of being particularly cautious when checking the correct adaptation of these children to the orthosis, according to their growth progress, and perhaps a more restricted or monitored time not wearing those orthosis should be practiced, as these type of fractures have been previously described at night while the child is not wearing them [2], or even during physical treatment therapy, when orthosis may be removed to practice the exercises [13]. 


\section{Conclusion}

As it has been demonstrated with this case report and review of the published data, PF may develop complications that can go unnoticed due to the lack of symptoms. Not only because of the neuro-orthopedical condition of these patients, but also because of the likelihood of bone fractures, it seems to be clear that physicians who attend these patients should follow up them until their final growth, to slash possible complications and optimize the bone quality.

\section{References}

1. Akbar M (2010) Fractures in myelomeningocele. Journal of Orthopaedics and Traumatology 11(3): 175-182.

2. Lock TR, Aronson DD (1989) Fractures in patients who have myelomeningocele. J Bone Joint Surg Am 71(8): 1153-1157.

3. Aliatakis N (2020) Age-specific occurrence of pathological fractures in patients with spina bifida. European Journal of Pediatrics 179(5): 773779.

4. Gibbs JC (2015) Muscle density and bone quality of the distal lower extremity among individuals with chronic spinal cord injury. Topics in Spinal Cord Injury Rehabilitation 21(4): 282-293.

5. Kumar SJ, Cowell HR, Townsend P (1984) Physeal, metaphyseal, and diaphyseal injuries of the lower extremities in children with myelomeningocele. Journal of Pediatric Orthopedics 4(1): 25-27.
6. Cuxart A, Iborra J, Meléndez M, Pagès E (1992) Physeal injuries in myelomeningocele patients. Paraplegia 30(11): 791-794

7. Pfeil J, Fromm B, Carstens C, Cotta H (1990) Fractures and epiphyseal injuries in children with myelomeningocele. Zeitschrift fur Orthopadie und ihre Grenzgebiete 128(6): 551-558.

8. Townsend PF, Cowell HR, Steg NL (1979) Lower extremity fractures simulating infection in myelomeningocele. Clinical Orthopaedics And Related Research (144): 255-259.

9. Reikerås 0, Hellum C (1981) Fractures in children with myelomeningocele. Archives of orthopaedic and traumatic surgery 98(1): 25-28.

10. D’Andrea L, Catena N (2008) Femural shaft fracture in a newborn infant treated with axial external fixator: A Case Report. Journal of Pediatric Orthopaedics 28(1): 17-19.

11. Boyce AM, Tosi LL, Paul SM (2014) Bisphosphonate treatment for children with disabling conditions. PM \& R: The Journal of Injury, Function and Rehabilitation 6(5): 427-436.

12. Ooi HL, Briody J, McQuade M, Munns CF (2012) Zoledronic acid improves bone mineral density in pediatric spinal cord injury. Journal of Bone and Mineral Research 27(7): 1536-1540.

13. Della Grotta LM, Marine MB, Harris TL, Karmazyn B (2019) Classic metaphyseal lesion acquired during physical therapy. Clinical Imaging 54: 100-102. 